\title{
Transmission properties of hydrogenated amorphous silicon optical fibers into the mid-infrared regime
}

\author{
L. Shen ${ }^{1}$, N. Healy ${ }^{1}$, P. Mehta ${ }^{1}$, T. D. Day ${ }^{2}$, J. R. Sparks ${ }^{2}$, J. V. Badding ${ }^{2}$, \\ and A. C. Peacock ${ }^{1}$ \\ 1. Optoelectronics Research Centre, University of Southampton, Southampton SO17 1BJ, United Kingdom \\ 2. Department of Chemistry and Materials Research Institute, Pennsylvania State University 16802 PA, USA \\ acp@orc.soton.ac.uk
}

\begin{abstract}
The transmission properties of hydrogenated amorphous silicon core fibers are characterized from the near to mid-infrared regime. The low linear and nonlinear losses measured past $2 \mu \mathrm{m}$ indicate their suitability for applications in the life sciences.
\end{abstract}

(C) 2012 Optical Society of America

OCIS codes: $060.2270,060.2290,060.4370$.

\section{Introduction}

Hydrogenated amorphous silicon (a-Si:H) is becoming an increasingly popular material for nonlinear silicon photonics due to its low transmission losses, high Kerr nonlinearity and low fabrication costs [1]. To date, a number of important photonic functions have been demonstrated in a-Si:H waveguides in the near-infrared wavelength region including all-optical modulation, photon pair generation, and parametric amplification [2,3]. Although the large bandgap of the amorphous material $(\sim 1.7 \mathrm{eV})$ suggests that nonlinear absorption should be modest at telecoms wavelengths which are past the two-photon absorption (TPA) edge, in practice the reported values of the TPA parameter $\beta_{\text {TPA }}$ are of a similar magnitude to crystalline silicon [4]. In this paper we extend the characterization of our a-Si:H core fibers beyond the telecommunications window of $\sim 1.5 \mu \mathrm{m}$ and present the first measurements of the transmission properties into the mid-infrared regime of $2-3 \mu \mathrm{m}$. The low linear and nonlinear losses that are measured past $2 \mu \mathrm{m}$ suggest that these fibers could find applications in areas such as sensing, medicine and thermal imaging.

\section{Fabrication and Characterization}

Our silicon-core, silica-clad fibers are fabricated using the well-established high pressure microfluidic chemical technique [4]. In this process a mixture of silane and helium $\left(\mathrm{SiH}_{4} / \mathrm{He}\right)$ is passed through the central hole of the capillary under high pressures $\sim 35 \mathrm{MPa}$, whilst being heated in a tube furnace. By keeping the deposition temperatures below $450^{\circ} \mathrm{C}$, the dissociation of hydrogen from the precursor is not complete so that some remains in the core to passivate the dangling bonds, greatly reducing the optical transmission loss. The fiber used in our experiments was fabricated by depositing a-Si:H inside a $5.6 \mu \mathrm{m}$ silica capillary, as shown in the inset of Fig. 1(b). To ensure that the core material has sufficient hydrogen, micro-Raman measurements were undertaken using a HeNe laser at $633 \mathrm{~nm}$. The Raman spectrum plotted in Fig. 1(a) exhibits a strong broad peak around $480 \mathrm{~cm}^{-1}$ which is associated with the vibrational modes of amorphous silicon. The presence of hydrogen in the core material is confirmed by the observation of the $\mathrm{Si}-\mathrm{H}$ stretching mode seen in the inset at $\sim 2000 \mathrm{~cm}^{-1}$.
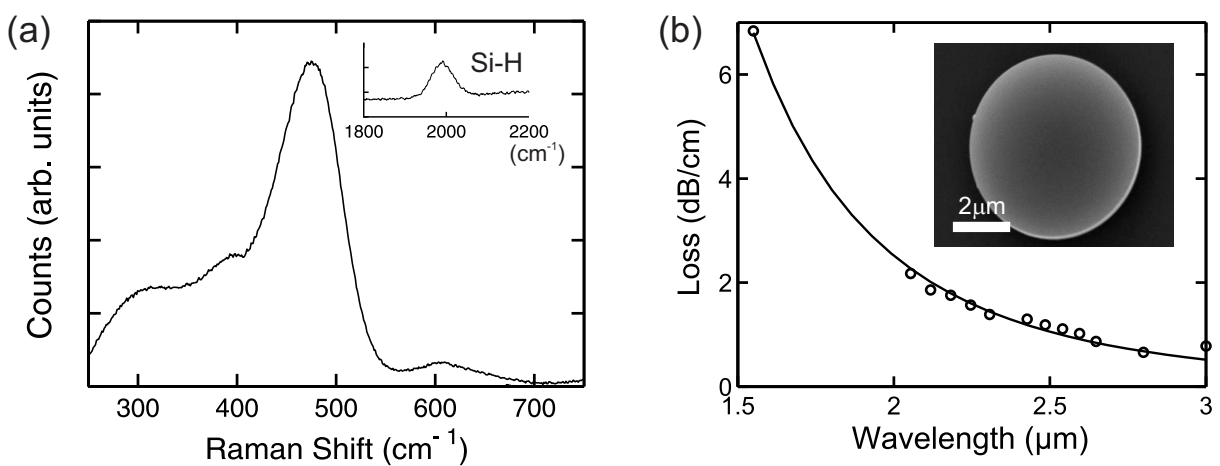

Fig. 1. (a) Raman spectrum of the amorphous material; inset, Si-H vibrational bond. (b) Linear losses as a function of wavelength together with a $\lambda^{-4}$ fit. Inset, SEM image of the a-Si:H core. 
To characterize the linear losses from the near to mid-infrared $(1.55-3 \mu \mathrm{m})$ a series of low power transmission measurements were conducted using three continuous wave laser sources: (i) a $1.55 \mu$ m diode, (ii) a tunable $\mathrm{Cr}^{+}: \mathrm{ZnSe}$ laser and (iii) an Aculight OPO. In both cases the light was launched into the silicon core via free space coupling using a $40 \times$ microscope objective lens and a second $40 \times$ objective was used to capture the transmitted light and focus it onto a power meter. The cutback technique was used to quantify the linear transmission losses as plotted in Fig. 1(b). These results follow the same $\lambda^{-4}$ trend of decreasing loss for increasing wavelength observed in our earlier silicon fibers, which we have previously attributed to scattering losses. The loss determined at $2.8 \mu \mathrm{m}(\sim 0.7 \mathrm{~dB} / \mathrm{cm})$ is one of the lowest values recorded in this wavelength regime, and we believe that this can be decreased with further optimization of the deposition process as the $6.7 \mathrm{~dB} / \mathrm{cm}$ value of the loss at $1.55 \mu \mathrm{m}$ is far from our lowest reported values of $\sim 1 \mathrm{~dB} / \mathrm{cm}[2]$.
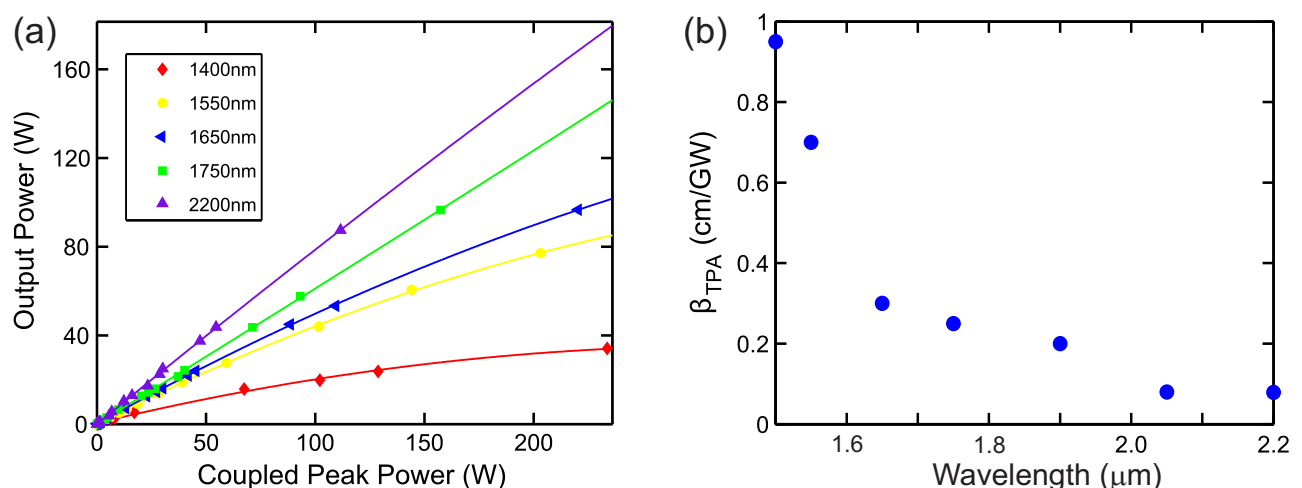

Fig. 2. (a) Nonlinear absorption measurements for the wavelengths given in the legend. (b) TPA parameter as a function of wavelength extracted from (a).

As the precise position of the TPA edge is not known for our material, nonlinear absorption measurements were performed over a range of wavelengths spanning half the theoretical bandgap energy of a-Si: $\mathrm{H}(\sim 0.85 \mathrm{eV})$ and into the mid-infrared. To obtain the high peak powers necessary to probe the nonlinear absorption, in these experiments we used a Ti:sapphire pumped OPO with $200 \mathrm{fs}$ pulses at $80 \mathrm{MHz}$. Fig. 2(a) plots the output power as a function of the coupled input power, clearly showing the onset of nonlinear absorption for wavelengths in the range $1.4-1.65 \mu \mathrm{m}$. The linear trend exhibited for the longer wavelengths where TPA is negligible, indicates the bandgap energy of our core material is likely to be in the region $1.4-1.5 \mathrm{eV}$. To establish the corresponding values of the TPA parameter, we follow the method outlined in Ref. [1] and obtain $\beta_{\mathrm{TPA}}$ from the slope of the inverse of the transmission given by $1 / T=P_{\text {in }} / P_{\text {out }}$. Plotting these values as a function of wavelength in Fig. 2(b), we find that $\beta_{\mathrm{TPA}}$ decreases rapidly over the wavelength range $1.5-1.65 \mu \mathrm{m}$, with $\beta_{\mathrm{TPA}}=0.7 \pm 0.08 \mathrm{~cm} / \mathrm{GW}$ at $1.55 \mu \mathrm{m}$, before reaching a plateau at an essentially negligible value of $0.08 \mathrm{~cm} / \mathrm{GW}$ for wavelengths $>2 \mu \mathrm{m}$. We anticipate that the size of the bandgap, and thus the position of the TPA edge and the values of $\beta_{\text {TPA }}$ can be tuned through the material deposition parameters to shift the region of low nonlinear loss to shorter wavelengths.

\section{Conclusion}

We have characterized the transmission properties of hydrogenated amorphous silicon fibers extending into the midinfrared, demonstrating some of the lowest losses in silicon in this wavelength region to date. The low nonlinear losses measured in these fibers at wavelengths $>2 \mu \mathrm{m}$ make them a suitable platform for applications in the life sciences.

\section{References}

1. B. Kuyken, H. Ji, S. Clemmen, S. K. Selvaraja, H. Hu, M. Pu, M. Galili, P. Jeppesen, G. Morthier, S. Massar, L.K. Oxenlwe, G. Roelkens, and R. Baets, "Nonlinear properties of and nonlinear processing in hydrogenated amorphous silicon waveguides," Opt. Express 19, B146-B153 (2011).

2. P. Mehta, N. Healy, T. D. Day, J. R. Sparks, P. J. A. Sazio, J. V. Badding, and A. C. Peacock, "All-optical modulation using two-photon absorption in silicon core optical fibers," Opt. Express 19, 19078-19083 (2011).

3. B. Kuyken, S. Clemmen, S. K. Selvaraja, W. Bogaerts, D. van Thourhout, P. Emplit, S. Massar, G. Roelkens, and R. Baets, "On-chip parametric amplification with $26.5 \mathrm{~dB}$ gain at telecommunications wavelengths using CMOS-compatible hydrogenated amorphous silicon waveguides," Opt. Lett. 36, 552-554 (2011).

4. P. Mehta, N. Healy, N. F. Baril, P. J. A. Sazio, J. V. Badding, and A. C. Peacock, "Nonlinear transmission properties of hydrogenated amorphous silicon core optical fibers,” Opt. Express 16, 16826-16831 (2010). 